\title{
Study of Lipid Profile in Newly Diagnosed Hypertensive Patients
}

\author{
MAK Akanda ${ }^{1}$, KN Choudhury $^{2}$, MZ Ali $^{1}$, LA Sayami ${ }^{1}$, RM Huda $^{1}$ \\ ${ }^{1}$ Department of Cardiology, NICVD, Dhaka, Department of Epidemiology, ${ }^{2}$ NCCRF\&HD, Dhaka
}

Key words: Hypertension, lipid profile, Total cholesterol, Triglyceride, $L D L c, H D L c$

\begin{abstract}
Abstret
Back ground: Hypertension is one of the most important modifiable risk factors for cardiovascular disease (CVD) and stroke. Dyslipidemia is closely associated with hypertension. Dyslipidemia and hypertension are the commonest risk factors for CVD. The aim of the present study was to compare different lipid parameters among newly diagnosed hypertensive patients with normotensive subjects in Bangladesh and find out the relationship.

Methods: This study was a cross sectional study in which 42 newly diagnosed hypertensive patients who were on no antihypertensive medication and 42 subjects with normal blood pressure (normotensive) were enrolled for compare. Lipid parameters total cholesterol (TC), triglyceride (TG), low density lipoprotein ( LDLc) and high density lipoprotein (HDLc) were estimated by Enzymatic colorimetric test.

Results: The mean systolic blood pressure of hypertensive and normotensive were $154.6 \pm 22.5$ vs $111.50 \pm 3.42$ mmof.Hg and mean diastolic blood pressure were $93.2 \pm 5.20$ vs $71.44 \pm 3.21 \mathrm{~mm}$ of $\mathrm{Hg}$. The mean of SBP and DBP of hypertensive patients was found to be higher than normotensive $(p<0.05)$.

There was significant increase in different lipid levels namely TC 199.4 $444.5 \mathrm{vs} 188.7 \pm 37.9 \mathrm{mg} / \mathrm{dl}$,

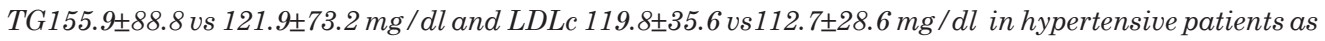
compared to normotensive $(p<0.05)$. However there was no significant difference in HDLc level. BMI and waist circumference showed significant association in hypertensive patients $(p<0.001)$.

Conclusion: Analytical results of the study revealed that hypertensive patients have been found to have close association with dyslipidemia, BMI and waist circumference. Hypertension and dyslipidemia can be modified either by proper life style changes or medical management or by the combination of the both. This study suggests that hypertensive patients need measurement of blood pressure and lipid profile at regular interval to prevent heart diseases and stroke.
\end{abstract}

(Cardiovasc. j. 2014; 6(2): 112-115)

\section{Introduction:}

Epidemiological studies have established a strong association between hypertension and cardiovascular disease (CVD). Hypertension is a major independent risk factor for the development of CVD, stroke \&renal failure. ${ }^{1}$ Dyslipidemia and hypertension are the commonest risk factors for coronary artery disease (CAD). ${ }^{2}$ Hypertensive persons have usually higher levels of serum lipids than normotensive persons. ${ }^{3}$ The changes in serum lipid profile levels should be actively investigated but a few studies have established relation between hypertension and dyslipidemia.

Atherosclerotic diseases are a leading cause of disability and death worldwide and two third of these are associated with dyslipidemia. Worldwide, there is a wide variation in mean population lipid profiles levels. Increased serum total cholesterol (TC), triglyceride (TG), low density lipoprotein (LDL-c) level and decreased high density lipoprotein (HDL-c) are associated major risk factors for cardiovascular disease (CVD). ${ }^{4}$

The Seventh report of the Joint National Committee on Prevention, Detection, Evaluation and Treatment of High Blood Pressure (JNC-7) classifies BP as normal (Systolic BP $<120 \mathrm{mmHg}$ and Diastolic $\mathrm{BP}<80 \mathrm{mmHg}$ ). Pre hypertension (Systolic BP 120-139mmHg and Diastolic BP 80$89 \mathrm{mmHg}$ ), hypertension stage I (Systolic BP 140$159 \mathrm{mmHg}$ and Diastolic BP $90-99 \mathrm{mmHg}$ and hypertension stage 2 (Systolic BP $160 \mathrm{mmHg}$ and Diastolic BP $100 \mathrm{mmHg}$ ) respectively. ${ }^{1}$

Overweight and obesity with increasing body mass index and waist circumference leads to adverse metabolic effects on blood pressure,

Address of Correspondence: Dr Md Abdul Kader Akanda, Department of Cardiology, NICVD, Dhaka, Bangladesh. E-mail: abdulkaderakanda@yahoo.com 
cholesterol and triglyceride level. Obesity and over weight is associated with increased risk of various disorders including dyslipidemia.

Various studies showed an association between BMI, dyslipidemia and hypertension. However, due to factors like socio-economic, geographic conditions, food habits, age sex, genetic and others influences these parameters. Therefore, this study was conceived with the aim of estimating serum lipid profile among newly diagnosed hypertensive patients with normotensive subjects and find out the relationship in Bangladesh.

\section{Methods:}

This study was a cross sectional study in which 42 newly diagnosed hypertensive patients who were on no antihypertensive medication and 42 subjects with normal blood pressure (normotensive) were enrolled for compare. These patients sought a through health cheek up including blood pressure assessment between January to December 2012 in National centre for control of Rheumatic fever and heart disease (NCCRF\&HD). All the subjects were residents of the surrounding areas and aged 30-60 years. Patients with features of any cardiac or renal complication and major medical problem were excluded. After obtaining informed consent data collectors completed data sheet by interview, clinical examination, anthropometric measurement and investigation.

\section{Blood Pressure measurement:}

Blood pressure was measured after the subject had reexamined for at least 5 minutes from right arm placed at the heart level by a physician. Two measurements were taken by a mercury sphygmomanometer with at least 5 minutes between successive measurements. The mean of two measurements of Korotkoff phase I was recorded for systolic blood pressure (SBP). The mean of two values of korotkoff phase IV was recorded for diastolic pressure (DBP). Hypertension was defined as an average SBP $>140 \mathrm{mmHg}$ and DBP >90 $\mathrm{mmHg}$ without antihypertensive medication. ${ }^{1}$

\section{Serum assessments:}

Venous blood was collected in the morning after an overnight fast and serum was used for the biochemical tests. Lipid parameters (TC, TG, LDLc and HDLc) were estimated by Enzymatic colorimetric test. Dyslipidemia was defined according to ATPIII report. Hypercholesterolemia was defined as fasting total serum cholesterol and triglyceride of greater than or equal to $200 \mathrm{mg} / \mathrm{dl}$ and 150 respectively. Blood concentration of LDL-C (low-density lipoprotein cholesterol) equal or above $150 \mathrm{mg} / \mathrm{dl}$ and blood concentration of HDL-C (high-density liprotein cholesterol) under $40 \mathrm{mg} / \mathrm{dl}$ respectively, were considered to be undesirable. ${ }^{5}$

\section{Statistical analysis}

The collected data were analyzed by SPSS software version 17. All values were expressed as mean and SD. Statistical significance of difference between cases and control groups were evaluated by student's "t" test and $p$-value $<0.05$ was considered as significant.

\section{Results:}

The mean systolic blood pressure (SBP) of hypertensive patients was $154.6 \pm 22.5 \mathrm{mmof} \mathrm{Hg}$ and that of normotensive subjects was111.5 \pm 3.42 $\mathrm{mm}$ of $\mathrm{Hg}$. The mean systolic blood pressure was found to be higher in hypertensive than normotensive $(p<0.05)$. Mean diastolic blood pressure of hypertensive cases was $95.2 \pm 5.20 \mathrm{~mm}$ of $\mathrm{Hg}$ and that of controls was $71.4 \pm 3.21 \mathrm{mmof}$ $\mathrm{Hg}$. The mean DBP of hypertensive cases was found to be higher than controls $(\mathrm{p}<0.05)$. (The results are shown in Table I and Fig 1)

\section{Table-I}

Comparison of Mean SBP and DBP between hypertensive and normotensive study population.

\begin{tabular}{|c|c|c|c|c|c|}
\hline \multirow{2}{*}{$\begin{array}{l}\text { Variables } \\
(\mathrm{mm} \text { of } \mathrm{Hg})\end{array}$} & \multicolumn{2}{|c|}{ Hypertensive } & \multicolumn{2}{|c|}{ Normotensive } & \multirow{2}{*}{$\begin{array}{c}\mathrm{P}- \\
\text { Value }\end{array}$} \\
\hline & Mean & $\mathrm{SD}$ & Mean & $\mathrm{SD}$ & \\
\hline $\begin{array}{l}\text { Systolic Blood } \\
\text { Pressure }\end{array}$ & 154.6 & 22.5 & 111.5 & 3.42 & 0.05 \\
\hline $\begin{array}{l}\text { Diastolic Blood } \\
\text { Pressure }\end{array}$ & 95.2 & 5.2 & 71.4 & 3.21 & 0.05 \\
\hline
\end{tabular}




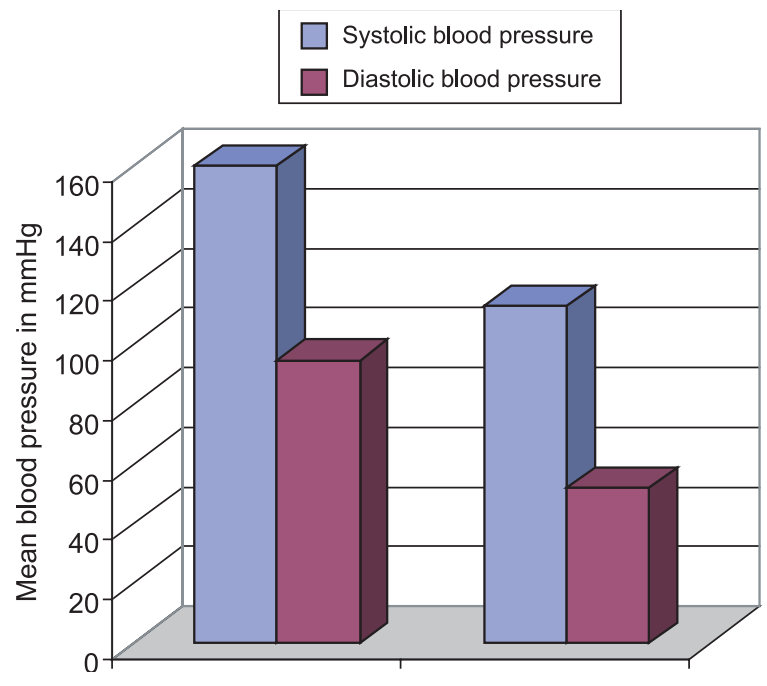

Fig.-1: Comparison and mean values of SBP and $D B P$ between hypertensive and normotensive study population.

The mean age of hypertensive and normotensive were $57.1 \pm 13.4$ vs $48.1 \pm 12.8$ years respectively. The mean total cholesterol (TC) in hypertensive was $199.4 \pm 44.5 \mathrm{mg} / \mathrm{dl}$ whereas that of normotensive was $188.7 \pm 37.9 \mathrm{mg} / \mathrm{dl}$. The mean of hypertensive was higher than normotensive $(p<0.05)$. The mean HDL of hypertensive was $35.43 \pm 4.21 \mathrm{mg} / \mathrm{dl}$ and normotensive was $36.21 \pm 5.51 \mathrm{mg} / \mathrm{dl}$ and it was not statistically significant. Mean LDL of hypertensive was $119.8 \pm 35.6 \mathrm{mg} / \mathrm{dl}$ and that of normotensive was $112.7 \pm 28.6 \mathrm{mg} / \mathrm{dl}$. The mean LDL of hypertensive cases was higher than that of normotensive $(p<0.05)$. The mean triglycerides of hypertensive and normotensive was $155.9 \pm 88.8$ vs $121.9 \pm 73.2 \mathrm{mg} /$ $\mathrm{dl}$ respectively and statistically significant $(\mathrm{p}<0.05)$. BMI and waist circumference showed significant association in hypertensive patients $(p<0.001)$. (The results are shown in Table II and Fig 2).

\section{Table-II}

Comparison of Mean lipid profile between hypertensive and normotensive study population.

\begin{tabular}{lcccccc}
\hline Indicators & \multicolumn{2}{c}{ Hypertensive } & & \multicolumn{2}{c}{ Normotensive } & P-Value \\
\cline { 2 - 3 } & Mean & SD & & Mean & SD & \\
\hline Age (in years) & 57.1 & 13.4 & & 48.12 .8 & 12.8 & $<0.001$ \\
Total cholesterol & 199.4 & 44.5 & 188.73 & 7.9 & $<0.001$ \\
HDLc & 35.43 & 4.21 & 36.21 & 5.51 & 0.195 \\
LDLc & 119.8 & 35.6 & 112.7 & 28.6 & $<0.001$ \\
Triglyceride & 155.9 & 88.8 & 121.9 & 73.2 & $<0.001$ \\
BMI & 26.3 & 2.3 & 25.8 & 4.3 & $<0.001$ \\
Waist Circumference 86.1 & 8.9 & 78.27 & 5.3 & $<0.001$ \\
\hline
\end{tabular}

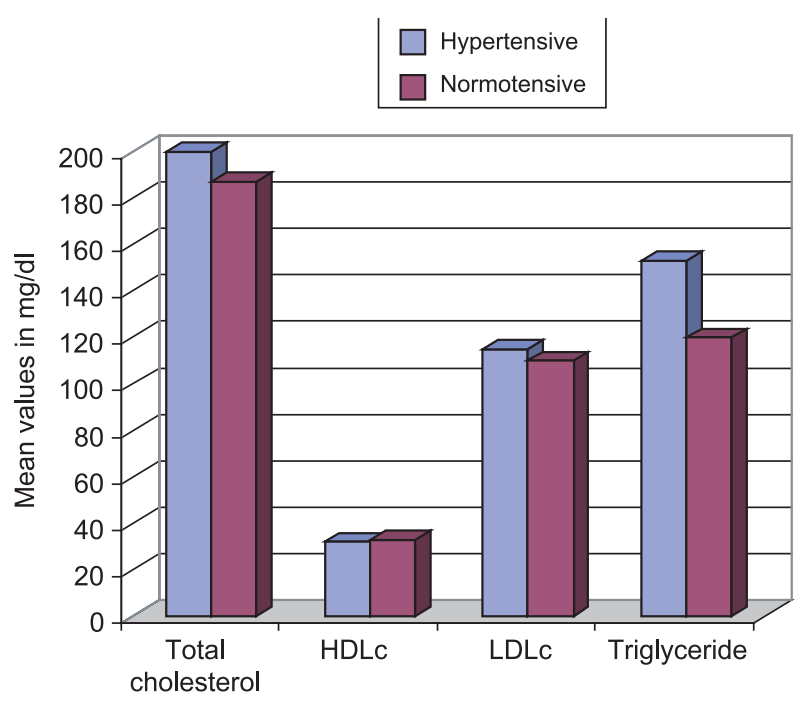

Fig.-2: Comparison of mean levels for TC, HDLc, $L D L c$ and $T G$ between hypertensive and normotensive study population.

\section{Discussion:}

Hypertension is recognized globally as a major public health problem. ${ }^{6}$ It is known as the well known risk factor for coronary heart disease, type 2 diabetes mellitus and renal diseases. ${ }^{7}$ About $80 \%$ of hypertensive persons have co morbidities such as obesity, glucose intolerance, low HDL-Cho, high LDL-Cho and increased triglycerides etc. Two or more co morbidities are found in about more than 50\% hypertensive patients. Present study was focused on to study the lipid profile pattern of hypertensive patients to normotensive. In present study the results revealed that the mean value of serum total cholesterol, triglycerides and serum LDLcholesterol was significantly higher and statistically significant. HDL-cholesterol levels were similar both the hypertensive and normotensive. A prospective study which is based in the northern region of Bangladesh, to investigate the lipid profile status in hypertensive patients as compared to healthy normotensive controls. Their study revealed similar findings of high serum total cholesterol, triglycerides and LDL-cholesterol as observed in our study. ${ }^{8}$ Our findings of increased level of total cholesterol in hypertensive patients are similar to the findings of some other studies. ${ }^{9} \mathrm{~A}$ prospective study conducted in Bangladesh on type 2 diabetes mellitus patients with and without hypertension revealed significantly high 
total cholesterol, triglycerides and LDLcholesterol in hypertensive patients with type 2 diabetes mellitus as compared to the normotensive type 2 diabetes mellitus subjects. 10 Few studies showed strong association of hypertension and dyslipidimia with major risk factors of coronary heart disease. ${ }^{11} \mathrm{BMI}$ and waist circumference showed significant association in hypertensive patients in our study. Similar findings showed in association of lipid profile and body mass index in hypertensive patients of Eastern Nepal. ${ }^{12}$

Based on the results obtained from the present study, we concluded that serum lipid profile especially total cholesterol, triglycerides and LDL-cholesterol levels are positively associated with hypertension.

\section{Conclusion:}

Analytical results of the study revealed that hypertensive patients have been found to have close association with dyslipidemia. Hypertension and dyslipidemia can be modified either by proper life style changes or medical management or by the combination of the both. This study suggests that hypertensive patients need measurement of blood pressure and lipid profile at regular interval to prevent heart diseases and stroke.

\section{Acknowledgment:}

Authors are very grateful to all the participants for the study. The authors felt immense thanks to physicians, pathologists and pathological staff of National Centre for Control of Rheumatic fever and Heart Disease.

Conflict of Interest - None.

\section{Reference:}

1. Treatment of hypertension in the prevention and management of Ischemic heart disease: A scientific statement from the American heart association council for high blood pressure research and councils on clinical cardiology and epidemiology and prevention. J Hypertens 2007; 115: 2761-2788.

2. Lakhsmankumar N, Deepthi J, Rao YN and Kiran Deedi M. Study of lipid profile, serum magnesium and blood glucose in hypertension. Biology and Medicine 2010; 2(1):6-16.

3. Lipid Research Clinic Programme: The lipid research clinics, coronary primary prevention trial results -2. JAMA 1984; 251:365-374.

4. Caroll MD, Lachev DA, Sorlie PD, et al. Trends in serum lipids and lipoproteins of adults. JAMA 2005; 294:17731781.

5. Expert Panel on Detection: Evaluation and Treatment of High Blood Cholesterol in Adults. Executive Summary of the Third Report of the National Cholesterol Education Program (NCEP) Expert Panel on Detection, Evaluation and Treatment of High Blood Cholesterol in Adults (Adults Treatment Panel III). JAMA 2011; 185(19):2486-2497.

6. Cappuccio FP, Micah FB, Emmett L. Prevalence, detection, management and control of hypertension in Ashanti, West Afric. Hypertension 2004; 43:1017-1022.

7. Gupta R. Trends in hypertension epidemiology in India. $J$ Hum Hypertens 2004; 18:73-78.

8. Ms Saha, Shaha NK, et al. Serum lipid profile of hypertensive patients in northern region of Bangladesh. J Bioscience 2006; 14: 93-97.

9. Shahadat H, Maliha R, et al. Study of Serum lipid profile in essential hypertensive patients. Mymensingh Med $J$ 1999; 8(1):22-25.

10. Alam SM, Ali S, Deb K, et al. Serum lipid profile in hypertensive and normotensive type 2 diabetes mellitus patients- a comparative study. Mymensingh Med $J$ 2003; 1:13-16.

11. Joseph Osagie Idemudia, Emmanuel Ike, et al. Plasma lipid profiles in hypertensives Nigerians. The Internet Journal of Cardiovascular Research. ISSN: 1540-2592. 6(2). DOI:105580/117f.

12. Baral N, Jha P, Sridhar MG, et al. Association of lipid profile and Body mass index in hypertensive patients of Eastern Nepal. J Nepal Med Assoc 2006; 45: 306-309. 